\title{
Polarization of Dipole Radiation in Quantum Domain
}

\author{
Alexander S. Shumovsky and Özgür E. Müstecaplığlu \\ Physics Department, Bilkent University, Bilkent, 06533 Ankara, Turkey
}

(Received 2 October 1997)

\begin{abstract}
For a dipole radiation, the set of generalized Stokes parameters and corresponding Stokes operators are discussed. A qualitatively new behavior of quantum fluctuations of the Stokes parameters is predicted. The possibility to check this behavior in the eight-port operational measurement is shown. [S0031-9007(98)05314-9]
\end{abstract}

PACS numbers: 42.50.Dv, 42.25.Ja, 42.50.Lc

The polarization properties of a classical radiation are usually specified by the set of Stokes parameters [1] determined for a transverse field either in the linear polarization basis or in the circular polarization basis. The quantum counterpart is provided by the Stokes operators which can be obtained from the Stokes parameters by standard quantization of the field amplitudes [2,3]. Let us stress here that within the quantum optics, describing the radiation as a beam of photons, the polarization should be determined as a given spin state of photons, forming the beam. Spin of a photon is defined as the minimum value of the angular momentum and is equal to 1 (e.g., see [4]). Thus, it has three projections and therefore just three spin states and corresponding polarizations should be taken into consideration.

An example is provided by a dipole radiation when, due to the selection rules, the photons with the angular momentum 1 are emitted. It is well known that even in the classical picture, the dipole radiation always has a longitudinal component in addition to the transversal components [5]. Since this component decays with the distance quite rapidly, it is neglected in the far zone where the standard Stokes parameters for a completely transverse field are determined. Thus, the conventional definition of the Stokes parameters of the dipole radiation should be considered as an approximation which is known to be valid in the far zone. However, it is not a case in the quantum domain where one cannot neglect the longitudinal component a priori. Actually, even in the far zone, where the longitudinal component with the projection of spin $m=0$ contains very few photons and could be approximated by the vacuum state, it may contribute into the quantum fluctuations of different physical parameters. Therefore, it seems to be important to estimate the contribution of the longitudinal component with no resort to the transverse field approximation.

In view of this aim, let us consider the classical tensor of polarization [6] with the components which are slowly varying bilinear forms with respect to the complex electric field amplitudes $\vec{E}_{\lambda j m}$ where the index $\lambda$ shows the type of radiation (either electric or magnetic) and $j, m(|m| \leq j)$ are the indexes of the multipole expansion [5]. In the case of a dipole radiation $j=1, m=0, \pm 1$. The modes with $m= \pm 1$ correspond to the circularly polarized com- ponents with the opposite helicities while $m=0$ specifies the linearly polarized longitudinal component. Hence, the tensor of polarization has nine components and as usual it is represented as a superposition of a diagonal and Hermitian parts. Therefore, the polarization is specified by five real parameters. Actually, we have the intensities of three components and the phase differences between the components $\Delta_{m m^{\prime}}$ such that $\sum_{m} \Delta_{m m+1}=0$ as the independent parameters. Let us stress the difference with the case of a purely transversal field when the tensor of polarization has only four components and polarization is specified by three real parameters (two intensities and one phase difference).

It is now a straightforward matter to arrive at the following relations, determining the generalized Stokes parameters of the dipole radiation [7]:

$$
\begin{aligned}
& s_{0}=\sum_{m}\left|\vec{E}_{m}^{*} \cdot \vec{E}\right|^{2}, \\
& s_{1}=\operatorname{Re} \sum_{m}\left(\vec{E}_{m}^{*} \cdot \vec{E}\right)^{*}\left(\vec{E}_{m+1}^{*} \cdot \vec{E}\right), \\
& s_{2}=\operatorname{Im} \sum_{m}\left(\vec{E}_{m}^{*} \cdot \vec{E}\right)^{*}\left(\vec{E}_{m+1}^{*} \cdot \vec{E}\right), \\
& s_{3}=\left|\overrightarrow{E_{-}^{*}} \cdot \vec{E}\right|^{2}-\left|\vec{E}_{+}^{*} \cdot \vec{E}\right|^{2}, \\
& s_{4}=2\left|\vec{E}_{0}^{*} \cdot \vec{E}\right|^{2}-\left(\left|\vec{E}_{+}^{*} \cdot \vec{E}\right|^{2}+\left|\vec{E}_{-}^{*} \cdot \vec{E}\right|^{2}\right) .
\end{aligned}
$$

Here $m=0, \pm 1, \vec{E}_{m}=\vec{E}_{\lambda 1 m}$, and

$$
\begin{aligned}
\vec{E} & =\sum_{\lambda j m}\left(a_{\lambda j m} \vec{E}_{\lambda j m}+\text { c.c. }\right), \\
a_{\lambda j m} & =\int r^{2} d r d \Omega \vec{E}_{\lambda j m}^{*} \cdot \vec{E} .
\end{aligned}
$$

Then the generalized Stokes operators can be obtained from (1) by quantization of the field amplitudes in the representation of spherical photons [8] as follows:

$$
\begin{aligned}
& S_{0}=\sum_{m} \hat{a}_{m}^{+} \hat{a}_{m}, \\
& S_{1}=\frac{1}{2}\left[\left(\hat{a}_{+}^{+}+\hat{a}_{-}^{+}\right) \hat{a}_{0}+\hat{a}_{-}^{+} \hat{a}_{+}+\text {H.c. }\right], \\
& S_{2}=\frac{-i}{2}\left[\left(\hat{a}_{+}^{+}-\hat{a}_{-}^{+}\right) \hat{a}_{0}+\hat{a}_{-}^{+} \hat{a}_{+}-\text {H.c. }\right], \\
& S_{3}=\hat{a}_{-}^{+} \hat{a}_{-}-\hat{a}_{+}^{+} \hat{a}_{+}, \\
& S_{4}=2 \hat{a}_{0}^{+} \hat{a}_{0}-\left(\hat{a}_{+}^{+} \hat{a}_{+}+\hat{a}_{-}^{+} \hat{a}_{-}\right) .
\end{aligned}
$$


Here the operators $\hat{a}_{m}$ describe the dipole photons with the angular momentum $j=1$ and projection $m$.

To establish contact with the standard definitions of the Stokes parameters and operators, let us put $\left|\vec{E}_{0}\right|=0$ in (1). Under this assumption, Eqs. (1) formally coincide with the definition of ordinary Stokes parameters in the circular polarization basis (e.g., see [1,5]). Since the above assumption corresponds to the transverse wave approximation (in far zone), one can quantize the reduced Eqs. (1) with respect to the "plane photons" with given linear momentum to get the standard Stokes operators [2] of the form

$$
\begin{aligned}
& S_{0}=\hat{a}_{+}^{+} \hat{a}_{+}+\hat{a}_{-}^{+} \hat{a}_{-}, \\
& S_{1}=\frac{1}{2}\left(\hat{a}_{-}^{+} \hat{a}_{+}+\hat{a}_{+}^{+} \hat{a}_{-}\right), \\
& S_{2}=\frac{1}{2 i}\left(\hat{a}_{-}^{+} \hat{a}_{+}-\hat{a}_{+}^{+} \hat{a}_{-}\right), \\
& S_{3}=\hat{a}_{+}^{+} \hat{a}_{+}-\hat{a}_{-}^{+} \hat{a}_{-} .
\end{aligned}
$$

Here the operators $\hat{a}_{ \pm}=\left(\hat{a}_{x} \pm i \hat{a}_{y}\right) / \sqrt{2}$ describe the photons with two possible helicities in terms of the photons with two linear polarizations in the transverse field [3].

Let us compare the definitions (2) and (3). Suppose that the longitudinal component of the dipole radiation is in the vacuum state (far zone). Then the averaging with respect to the state of radiation field leads to the equalities

$$
\begin{aligned}
& \left\langle S_{0}\right\rangle=-\left\langle S_{4}\right\rangle=\left\langle S_{0}\right\rangle, \\
& \left\langle S_{k}\right\rangle=\left\langle S_{k}\right\rangle, \quad k=1,2,3,
\end{aligned}
$$

expressing the fact that the standard definition of the Stokes parameters follows from more general formulas (1). In a particular case of some considerable interest when both circularly polarized components are in the coherent states with one and the same intensity we get

$$
\begin{aligned}
& \left\langle S_{1}\right\rangle=|\alpha|^{2} \cos \Delta_{+-}, \\
& \left\langle S_{2}\right\rangle=|\alpha|^{2} \sin \Delta_{+-}, \\
& \left\langle S_{0}\right\rangle=2|\alpha|^{2}, \quad\left\langle S_{3}\right\rangle=0,
\end{aligned}
$$

where $\alpha_{ \pm}$is the parameter of corresponding coherent state, $\left|\alpha_{ \pm}\right| \equiv|\alpha|$, and $\Delta_{+-} \equiv \arg \alpha_{+}-\arg \alpha_{-}$.

At the same time, Eqs. (2) and (3) determine very different operator constructions. Although both sets consist of the Hermitian operators, they have different commutation relations, determining different possibilities of measurement. Since $\left[S_{1}, S_{2}\right] \neq 0$, corresponding Stokes parameters cannot be measured simultaneously. In view of the classical interpretation, it seems to be a bit strange. As a matter of fact, the Stokes parameters $\left\langle S_{1,2}\right\rangle$ determine the cosine and sine of the classical phase difference between two circularly polarized components [1]. This interpretation is consistent with Eqs. (5). Therefore, one could expect to have $S_{1}$ and $S_{2}$ as the commuting operators since they are the functions of one and the same argument.
On the contrary, $\left[S_{1}, S_{2}\right]=0$ and $\left[S_{1,2}, S_{0}\right]=0$ so that these three generalized Stokes parameters can be measured at once. Moreover, the operators $S_{1}, S_{2}$ are directly connected with the operators of cosine and sine of the azimuthal phase of angular momentum of the dipole radiation [7,9] and can be obtained from the conservation of the angular momentum in the process of radiation. The difference in the commutation properties can be traced in the most clear way in the quantum fluctuations of the Stokes parameters. Precisely, in the case of generalized Stokes operators (2) we get for the variance

$$
V\left(S_{1}\right)=|\alpha|^{2}\left(1+\frac{1}{2} \cos \Delta_{+-}\right),
$$

while $V\left(S_{1}\right)=|\alpha|^{2} / 2$. Thus, the quantum fluctuations of $S_{1}$ are much stronger than that for $S_{1}$. Moreover, they are qualitatively different because of the dependence on $\Delta_{+-}$in (6). A similar result can be obtained for $S_{2}$ and $S_{2}$ as well. Let us stress that the fluctuations of the total intensities $S_{0}$ and $S_{0}$ have the same magnitude in the case of the vacuum longitudinal field under consideration.

Thus, it is shown that the contribution of the longitudinal component of dipole radiation into the quantum fluctuations of the Stokes parameters is important even if this component is taken in the vacuum state when it does not contribute into the Stokes parameters per se. Because of the commutation properties of the operators (2), the eightport operational scheme $[10,11]$ might be used to detect the variances $V\left(S_{1,2}\right)$ to make sure of the $\Delta$ dependence predicted by Eq. (6). The above results are valid for both electric and magnetic dipole radiation. Similar results can be obtained for other multipole radiations.

One of the authors (A. S.) thanks Dr. V. Rupasov from the University of Toronto for stimulating discussions.

[1] M. Born and E. Wolf, Principles of Optics (Pergamon Press, Oxford, 1984).

[2] J. M. Jauch and F. Rohrlich, The Theory of Photons and Electrons (Addison-Wesley, Reading, MA, 1959).

[3] A. Luis and L. L. Sánchez-Soto, Phys. Rev. A 48, 4702 (1993).

[4] V.B. Berestetskii, E. M. Lifshitz, and L.P. Pitaevskii, Quantum Electrodynamics (Pergamon Press, Oxford, 1982).

[5] J.D. Jackson, Classical Electrodynamics (Wiley, New York, 1975).

[6] L.D. Landau and E. M. Lifshitz, Classical Theory of Fields (Pergamon Press, Oxford, 1971).

[7] A. S. Shumovsky and Ö. E. Müstecaplıŏlu, J. Mod. Opt. (to be published).

[8] W. Heitler, The Quantum Theory of Radiation (Dover, New York, 1984).

[9] A. S. Shumovsky, Opt. Commun. 136, 219 (1997).

[10] J. W. Noh, A. Fougéres, and L. Mandel, Phys. Rev. A 45, 242 (1992).

[11] T. Hakioğlu, A.S. Shumovsky, and O. Aytür, Phys. Lett. A 149, 304 (1994). 